\title{
The Sensorimotor System Can Sculpt Behaviorally Relevant Representations for Motor Learning
}

\author{
DDavid W. Franklin, ${ }^{1,2}{ }^{\circledR}$ Alexandra V. Batchelor, ${ }^{1,3}$ and ${ }^{\circledR}$ Daniel M. Wolpert ${ }^{1}$
}

\section{DOI:http://dx.doi.org/10.1523/ENEURO.0070-16.2016}

\begin{abstract}
${ }^{1}$ Computational and Biological Learning Laboratory, Department of Engineering, University of Cambridge, Cambridge CB2 1PZ, United Kingdom, ${ }^{2}$ Neuromuscular Diagnostics, Department of Sport and Health Sciences, Technical University of Munich, 80992 Munich, Germany, ${ }^{3}$ Department of Physiology, Development and Neuroscience, University of Cambridge, Cambridge CB2 3EG, United Kingdom
\end{abstract}

\begin{abstract}
The coordinate system in which humans learn novel motor skills is controversial. The representation of sensorimotor skills has been extensively studied by examining generalization after learning perturbations specifically designed to be ambiguous as to their coordinate system. Recent studies have found that learning is not represented in any simple coordinate system and can potentially be accounted for by a mixed representation. Here, instead of probing generalization, which has led to conflicting results, we examine whether novel dynamics can be learned when explicitly and unambiguously presented in particular coordinate systems. Subjects performed center-out reaches to targets in the presence of a force field, while varying the orientation of their hand (i.e., the wrist angle) across trials. Different groups of subjects experienced force fields that were explicitly presented either in Cartesian coordinates (field independent of hand orientation), in object coordinates (field rotated with hand orientation), or in anti-object coordinates (field rotated counter to hand orientation). Subjects learned to represent the dynamics when presented in either Cartesian or object coordinates, learning these as well as an ambiguous force field. However, learning was slower for the object-based dynamics and substantially impaired for the anti-object presentation. Our results show that the motor system is able to tune its representation to at least two natural coordinate systems but is impaired when the representation of the task does not correspond to a behaviorally relevant coordinate system. Our results show that the motor system can sculpt its representation through experience to match those of natural tasks.
\end{abstract}

Key words: coordinate frame; dynamics; motor learning

\section{Significance Statement}

The nature of the coordinate system in which humans learn motor skills has been highly controversial. Despite extensive experimental work, the results are highly conflicting (e.g., intrinsic joint-based vs Cartesian vs mixed coordinates). In our study, we show that the motor system is able to sculpt and tune its representation to at least two natural coordinate systems. Importantly, we also show that learning is impaired for a task of equal complexity that does not correspond to a naturalistic coordinate system. Our results suggest that the previously conflicting findings arise primarily from the use of novel skills that are ambiguous as to their coordinates, thereby making the experimental results highly sensitive to small differences in the experimental design. 


\section{Introduction}

The search for the coordinate system of motor control has been one of the holy grails of sensorimotor neuroscience at both the neural level (Kakei et al., 1999; Hatsopoulos, 2005; Aflalo and Graziano, 2007; Scott, 2008; Kalaska, 2009; Graziano, 2011; Churchland et al., 2012) and the behavioral level (Shadmehr and Mussa-Ivaldi, 1994; Malfait et al., 2005; Brayanov et al., 2012; Berniker et al., 2014), often with conflicting interpretations. Many studies have tried to identify the coordinate system in which motor skills are encoded by examining the generalization of learning. Although initial experiments suggested that the representation of novel dynamics occurs in simple intrinsic coordinates (i.e., in terms of joint torques and joint velocities; Shadmehr and Mussa-Ivaldi, 1994; Malfait et al., 2002, 2005), recent studies have questioned these results (Haswell et al., 2009; Brayanov et al., 2012; Berniker et al., 2014; Parmar et al., 2015). For example, Berniker et al. (2014) examined whether generalization could be accounted for by the sensorimotor system representing dynamics in intrinsic joint, extrinsic Cartesian, or object-based representations. They found that no single coordinate frame could explain the generalization of dynamics and that the representation was best accounted for by a mixture of these coordinate systems (Parmar et al., 2015). Such a mixed representation is in accord with studies of visuomotor learning, which has been shown to result from a mixed gain field encoding of intrinsic and extrinsic coordinates (Brayanov et al., 2012). Critically, all of these previous studies have used perturbations that are designed to be ambiguous as to the representation of the task (e.g., a skew-viscous curl force field in a small workspace or a force field for a single reach direction), and, therefore, these perturbations could in theory have multiple different representations. Therefore, the conflicting results may well reflect the participant's personal biases as well as small differences in the experimental conditions and perturbations.

Received March 31, 2016; accepted June 19, 2016; First published July 28, 2016.

The authors declare no competing financial interests.

Author contributions: Author contributions: D.W.F., A.V.B., and D.M.W. designed research; D.W.F. and A.V.B. performed research; D.W.F. analyzed data; D.W.F. and D.M.W. wrote the paper.

This research was supported by the Wellcome Trust (Grants WT091547MA and WT097803MA) and the Royal Society (Noreen Murray Professorship in Neurobiology to D.W.).

Acknowledgments: We thank J.N. Ingram and I.S. Howard for their work in setting up the vBOT robotic interface used in this study. We also thank Sae Franklin for recording data.

Correspondence should be addressed to David W. Franklin, Neuromuscular Diagnostics, Department of Sport and Health Sciences, Technical University of Munich, Campus D-Uptown München, Georg-Brauchle-Ring 60/62, Munich 80992, Germany. E-mail: david.franklin@tum.de.

A.V. Batchelor's present address: Department of Neurobiology, Harvard Medical School, 220 Longwood Avenue, Boston, MA 02115.

DOI:http://dx.doi.org/10.1523/ENEURO.0070-16.2016

Copyright (C) 2016 Franklin et al.

This is an open-access article distributed under the terms of the Creative Commons Attribution 4.0 International, which permits unrestricted use, distribution and reproduction in any medium provided that the original work is properly attributed.
Here we take a novel approach and, instead of probing generalization, we examine how well novel dynamics can be learned when the dynamics are explicitly and unambiguously presented in particular natural and unnatural coordinate systems. This allows us to test whether the motor system can learn to represent dynamics in specific different coordinate systems. That is, previous work has suggested that motor adaptation occurs in a mixture of coordinate frames (Brayanov et al., 2012; Berniker et al., 2014; Parmar et al., 2015). In particular, in the study by Berniker et al. (2014) ambiguous dynamics were presented to subjects to determine how such learning generalizes, and the results were consistent with subjects using a mixture of coordinate systems to learn the dynamics. The authors suggested that a specific skill could be learned by an appropriate combination of the coordinate system for that skill. Here we compare, for the first time, the learning of an ambiguous field and several nonambiguous fields. This allowed us to examine directly the extent to which the proposed mixed representation could be shaped to learn specific fields. By providing the dynamics unambiguously to the subjects, we determine whether the subjects can learn to use a specific coordinate frame, or, in other words, sculpt the particular coordinate system in which the task is learned.

Subjects performed center-out reaching movements to the same targets, but with three different hand orientations (neutral, flexed, and extended). The force field that we generated at the hand could be independent of these hand orientations, and therefore the experience was of an unambiguous Cartesian force field. Alternatively, we could vary the force field in real time with the hand orientation so that the force field either rotated with the hand, as if the force field was generated by a hand-held tool (natural object-based coordinates), or rotated counter to the hand (unnatural anti-object coordinates). Our results clearly show that the motor system can represent dynamics equally well in different natural coordinate systems (Cartesian and object), but not in an arbitrary coordinate system (anti-object), even when matched for complexity. This suggests that the motor system can sculpt different natural coordinate systems that are appropriate to task demands.

\section{Materials and Methods}

Forty naive subjects ( 17 male and 23 female) participated in the experiment (mean age, $25.9 \pm 5.8$ years), with 10 subjects assigned randomly to each of four groups. All subjects were right handed, according to the Edinburgh handedness inventory (Oldfield, 1971). Subjects gave informed consent, and the experiments were approved by the local ethics committee.

\section{Experimental setup}

Subjects were seated in a chair with their shoulders restrained by a harness. They made reaching movements with their right arm in the horizontal plane at $\sim 10 \mathrm{~cm}$ below their shoulder level, while the forearm was supported by an air sled. Subjects grasped the vertical handle of the robotic manipulandum (VBOT), which was used to generate the environmental dynamics (Howard et al., 

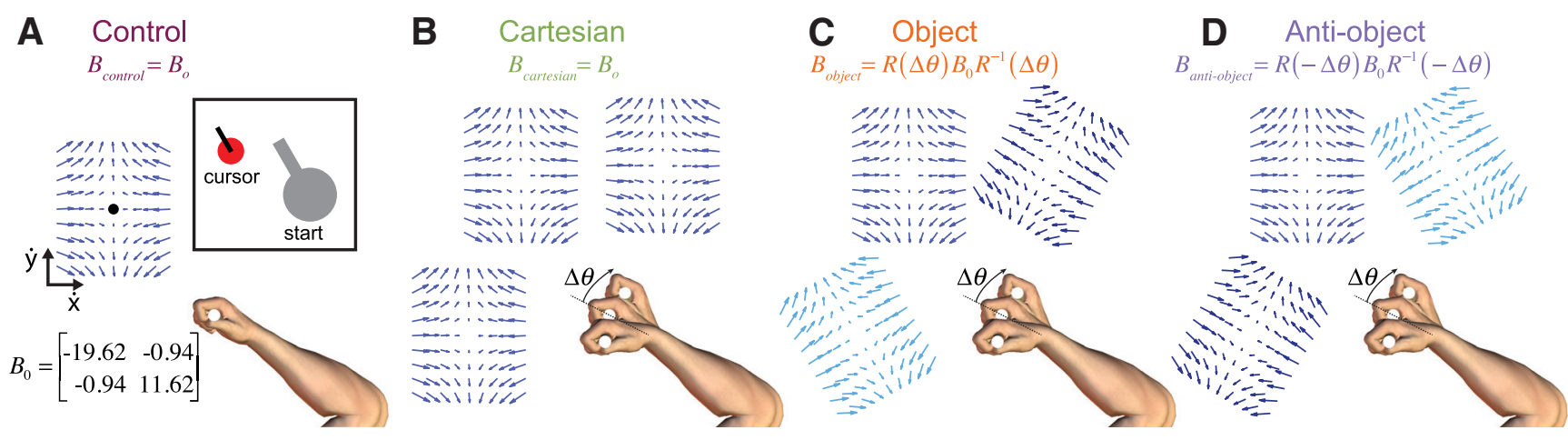

Figure 1. Experimental design and learning. $\boldsymbol{A}-\boldsymbol{D}$, Hand orientations and associated force fields, as force vectors experienced by the hand (zero velocity represented by the black circle) for the four groups of subjects. Note that the three vector fields are displayed separately, for clarity, but act over the same workspace. Similarly, the actual end point location of the hand for the three hand orientations is matched across the three conditions, but is separated here for clarity. Start location, targets, and cursor were displayed as a circle, showing position with a line indicating the orientation (inset).

2009). The handle was free to rotate around its vertical axis, and its position and orientation in extrinsic space was measured using optical encoders on the handle (58SA, IED) and motors. A fixed hand support was attached to the handle to ensure that the handle could only be grasped in a single way so that we could measure and control the hand orientation. End point forces at the handle were measured using an ATI Nano 25 six-axis forcetorque transducer (ATI Industrial Automation) mounted below the handle. The handle position and orientation, and the force data were sampled at $1 \mathrm{kHz}$. Visual feedback was provided using a computer monitor mounted above the VBOT and projected veridically to the subject via a mirror.

The hand cursor was displayed as a circular disk (0.5 $\mathrm{cm}$ radius) with a small bar emanating from the disk displaying the orientation of the handle (i.e., hand). Similarly, the start location and target were also displayed as circular disks ( $1.0 \mathrm{~cm}$ radius), each with a bar indicating the required hand orientation at the start and end of a movement (Fig. 1A, inset). On all trials, the start and target orientation were identical but could vary between trials. Prior to movement, the hand was required to be within the start location (positional tolerance, $0.5 \mathrm{~cm}$ ) and with orientation bars aligned (orientation tolerance $5^{\circ}$ ) for $750 \mathrm{~ms}$. A tone sounded to initiate the trial. Movements were made from the central start location to one of six possible targets equally spaced around a circle with a $10 \mathrm{~cm}$ radius. $A$ movement ended once the hand was at the target (positional tolerance, $0.8 \mathrm{~cm}$; orientation tolerance, $12.5^{\circ}$ ). Feedback was given as to whether the movement was too slow or fast, and if the peak speed was $50 \pm 8$ $\mathrm{cm} / \mathrm{s}$, a reward counter increased. The robot passively moved the hand back to the start position. Subjects took short breaks every 100 trials.

\section{Paradigm overview}

For the control group, all movements were made with the same hand orientation, whereas, for the other three groups, each movement was performed with three different hand orientations. The three hand orientations were a neutral position and $\pm 30^{\circ}$. The neutral orientation was with the hand slightly flexed, so that all postures could be comfortably reached. Initially, subjects performed 36 familiarization trials in a null field. Importantly, the end point location for movements in all three hand orientations were matched in Cartesian space. This means that for each set of hand orientations, the shoulder and elbow joint motions were slightly different.

The experiment was performed in two stages running consecutively, as follows: a pre-exposure phase (240 trials) was followed by the exposure phase (1320 trials). The pre-exposure phase was performed in a null field, while the exposure phase had a velocity-dependent force field. The exact nature of this force field varied between the experimental groups. Throughout the pre-exposure and exposure trials, blocks of trials were performed that included field trials (to all six targets) and channel trials (to four of the six targets). Channel trials were only used for the four targets in which significant lateral forces were applied by the force field. In the other two directions, a resistive force was produced, making channel trials uninformative (Fig. 2A). On a channel trial, the movement was confined to a simulated mechanical channel with a spring constant of $8000 \mathrm{~N} / \mathrm{m}$ and damping of $2 \mathrm{Ns} / \mathrm{m}$ (Scheidt et al., 2000; Milner and Franklin, 2005).

In the pre-exposure phase, each block (48 trials) consisted of 36 field trials ( 2 to each of the six targets with each of the three hand orientations) and 12 channel trials (1 to each of the four targets with each of the three hand orientations). In the exposure phase, each block (66 trials) consisted of 54 field trials ( 3 to each of the six targets with each of the three hand orientations) and 12 channel trials ( 1 to each of the four targets with each of the three hand orientations). Within a block, the order of the trials was pseudo-randomized while ensuring that two channel trials never occurred consecutively. A different pseudo-random sequence of trials was used for each subject.

\section{Control group}

For the control group, only the neutral hand orientation was used, and these subjects performed the same total 


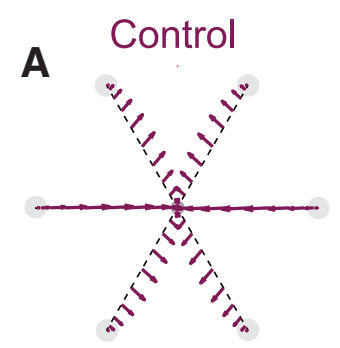

B

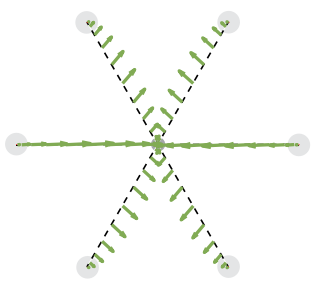

C
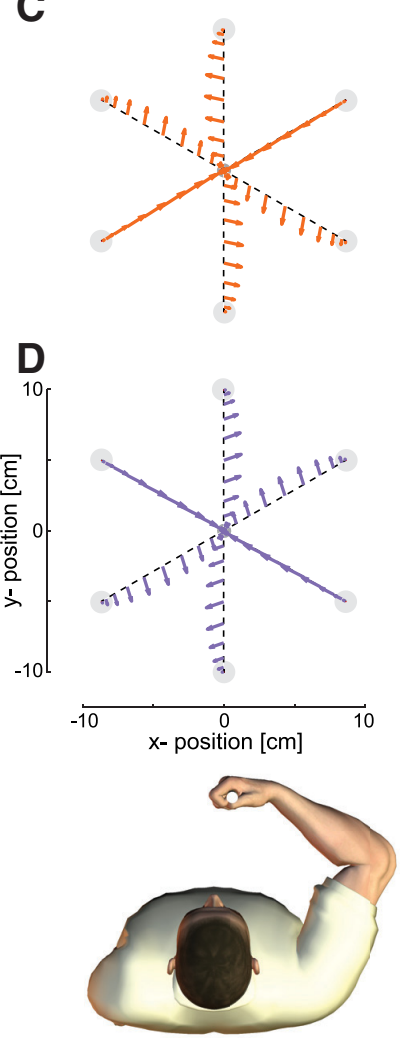

Cartesian
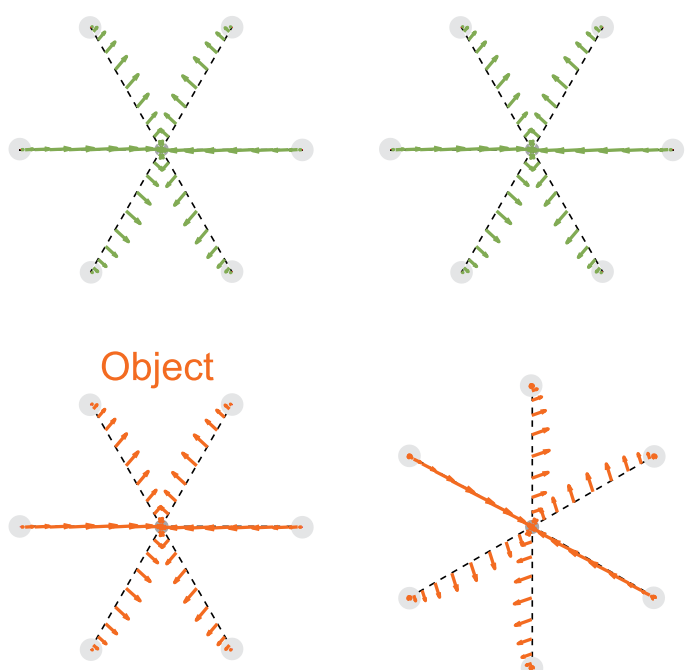

Anti-object
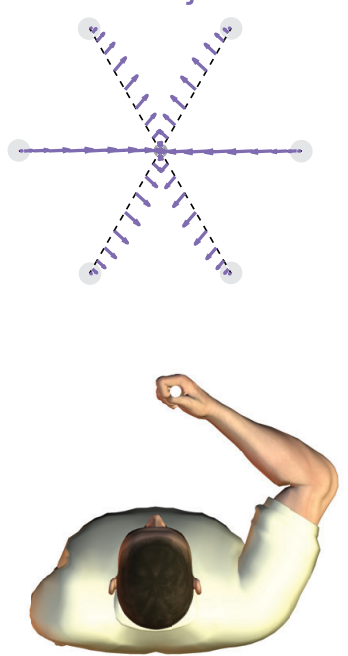
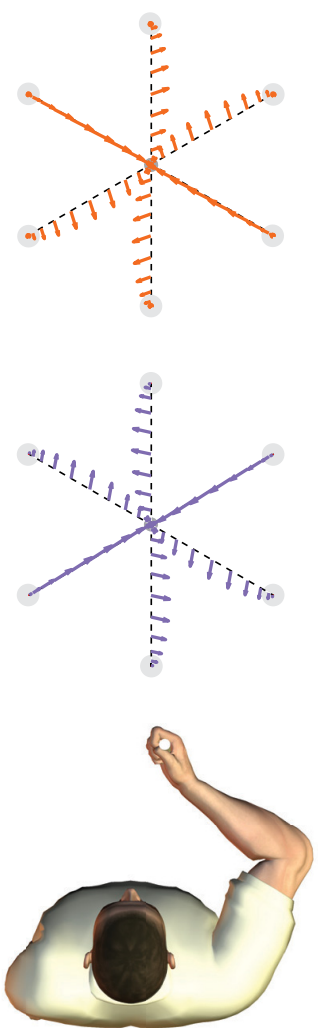

Figure 2. Force field conditions and movements across the experimental design. The wrist configuration (flexed, neutral, and extended) is shown for the three columns at the bottom of the figure. $\boldsymbol{A}$, The control group always performed movements in six directions with the neutral wrist configuration. $\boldsymbol{B}$, The Cartesian group performed reaching six reaching movements with the same Cartesian direction and forces for all three wrist orientations. $\boldsymbol{C}$, The object group. The six reaching directions were rotated for each wrist orientation such that the forces experienced were identical to the Cartesian condition (but rotated by $30^{\circ}$ ). $\boldsymbol{D}$, For the anti-object group, the force field and the reaching directions rotated in the opposite direction to the wrist. However, the forces experienced still matched those of the other groups. Importantly, the force fields and reaching directions on the middle wrist configuration were identical across all four groups. 
number of reaching movements as all other groups. This group performed reaching movements to the six targets, and the force field dynamics were given by the following:

$$
\begin{aligned}
& F=B \cdot \dot{x} \\
& B=\left[\begin{array}{cc}
-19.62 & -0.94 \\
-0.94 & 11.62
\end{array}\right]
\end{aligned}
$$

where $F$ is the vector of $x$ - and $y$-forces applied to the hand in $N$, and $\dot{x}$ is the vector of hand velocities in meters per second (Fig. 1, vector field shown as a function of hand velocity with stationary shown by the central dot). This skew-viscous force field resists movements in some direction, assists in others, and acts at an angle to the movement direction for yet other movement directions (Fig. 2A). Critically, unlike a viscous curl field, the skewviscous field has a clear orientation (e.g., the axis of movement that is purely resistive). As this group of subjects performed the movements with only one hand orientation (the neutral one), in common with previous studies, the coordinate system of the dynamics is ambiguous (Shadmehr and Mussa-Ivaldi, 1994; Berniker et al., 2014).

\section{Cartesian group}

The Cartesian group of subjects performed reaching movements to the six targets with three different hand orientations (18 total conditions). The six targets were equally spaced at $60^{\circ}$, and the location of the targets was maintained for all hand orientations. The forces experienced differed across the six movements but, critically, did not vary with the hand orientation, and therefore the field is unambiguously consistent with forces represented in a Cartesian frame (Figs. 1B, 2B ). Importantly, the end point location of the hand for the three hand orientation conditions is matched, meaning that the elbow and shoulder angles are slightly different for these conditions. However, the wrist angle (intrinsic coordinates) is very different for the three orientations, and the field is independent of this angle, meaning that the Cartesian representation is distinguishable from an intrinsic or joint-based representation.

\section{Object group}

The object group of subjects also performed reaching movements to six targets with the three hand orientations. The force field was implemented as follows:

$$
F=R B R^{-1} \dot{x}
$$

where $R$ is the rotation matrix that corresponds to the hand orientation and thereby rotates the force field by the same angle as the instantaneous hand orientation. Although subjects were required to maintain the orientation of the hand at the start and end of the moment, during each movement small variations from this orientation were tracked and used to update the force field on-line within a movement. In this manner, the force field rotates with the orientation of the hand, identical to the way in which the dynamics of a grasped object would rotate as the hand orientation changed (Fig. 1C). We chose to rotate the targets with the required hand orientation $\left( \pm 30^{\circ}\right)$ so as to ensure that the forces experienced in this condition were identical to the Cartesian condition with the exception that the association of the forces to the movement varied with the hand orientations (Fig. $2 \mathrm{C}$ ). This ensures the mathematical complexity to be constant across groups. The forces matched those of the Cartesian group for the same target directions for the neutral hand position. Therefore, for this group the dynamics are unambiguously consistent with forces that rotate with the hand orientation.

\section{Anti-object group}

The anti-object group of subjects performed reaching movements similar to those performed by the object group, with the exception that the force field rotated in the opposite direction to the rotation of the hand (Fig. 1D). This produced force fields that were identical to those of the object group, but that were associated with different hand orientations (Fig. 2D). Therefore, the mathematical complexity of this field is the same as that for the object group, but rotates in the opposite direction to the way in which a hand-held tool would behave. Again, the targets rotated with the desired hand orientation, and the forces matched those of the Cartesian group for the same target directions in the neutral hand position. Note that, as the targets are spaced at $60^{\circ}$ intervals, $\pm 30^{\circ}$ rotations of the targets are identical.

\section{Analysis}

Analysis of the experimental data was performed using Matlab R2015a. Individual trials were aligned on movement onset. For each nonchannel trial, the maximum perpendicular error (MPE) was calculated and used as a measure of the kinematic error. The MPE is the signed maximum perpendicular distance between the hand trajectory and the straight line between the start and end targets. The sign of the measurements for different movement directions and hand orientations was adjusted so that positive numbers reflected errors due to the perturbing force field. The end point forces were examined on the channel trials to further measure the amount of adaptation. The force produced by subjects into the wall of the simulated channel was integrated across the movement. To evaluate the degree of compensation (Smith et al., 2006), the measured force was divided by the amount of force that would be required for perfect compensation in the force field (calculated as the force field strength multiplied by the actual velocity on each trial). The values of the percentage force compensation throughout the experiment are based on the compensation required in the force field. Therefore, values in the null force field before learning (pre-exposure phase) should be close to zero.

We performed hypothesis-based planned comparisons and report uncorrected $p$ values to determine statistical significance $(p<0.05)$. ANOVAs were examined in SPSS (version 21) using the general linear model. Comparisons were made with main effects of epoch (pre-exposure, early exposure, or late exposure), force field condition (four levels), movement direction (six levels), and hand orientation (three levels), when appropriate. For the object 

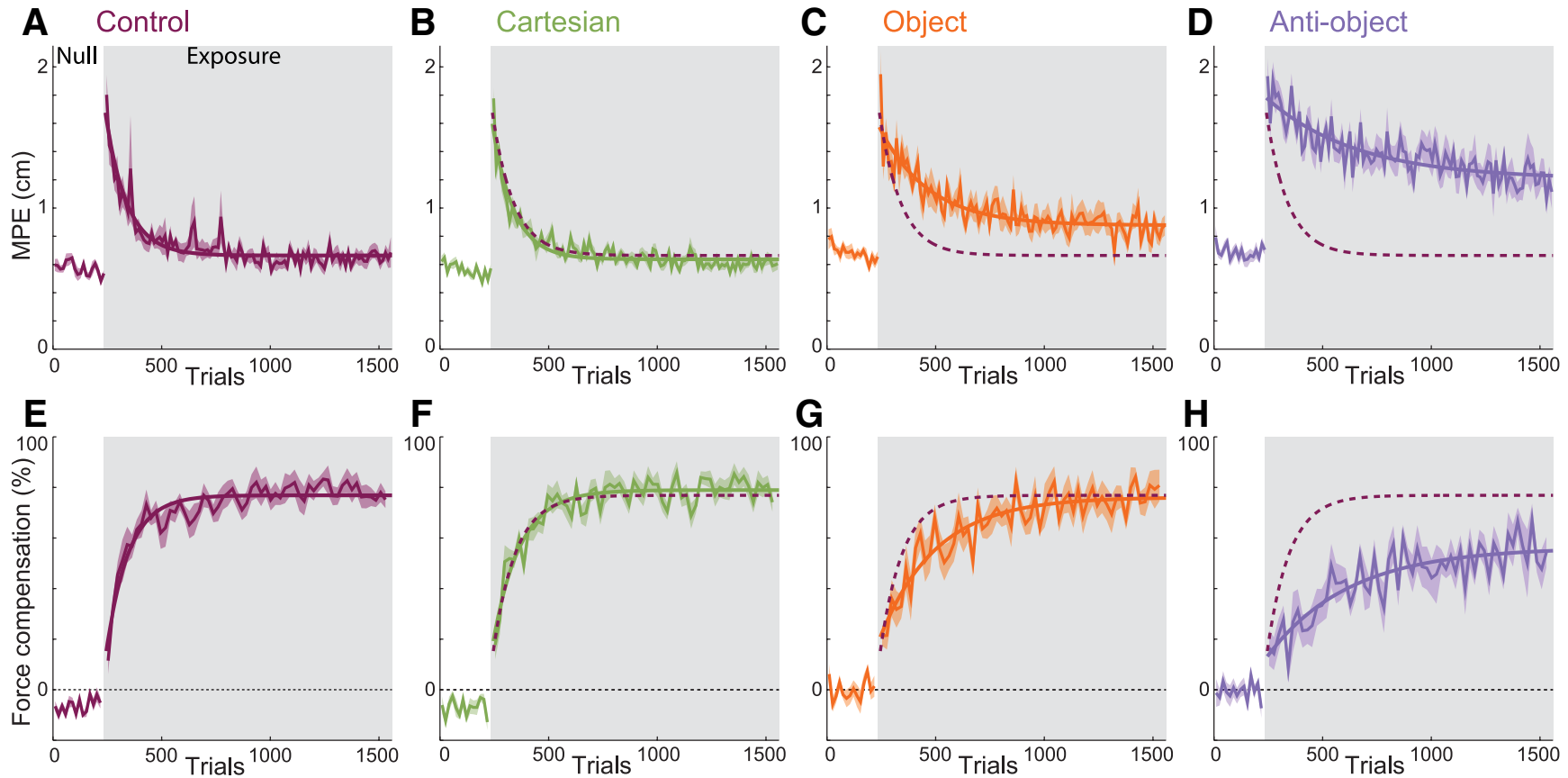

Figure 3. Adaptation to the force fields. $\boldsymbol{A}-\boldsymbol{D}$, Maximum perpendicular error. $\boldsymbol{E}-\boldsymbol{H}$, Force compensation percentage for the four groups (mean and SE across subjects) with exponential fits. Blocks of 10 nonchannel trials were used for the MPE, while blocks of four channel trials were used for the force compensation. The gray shaded region indicates the exposure period. The dashed curves are the exponential fit to the control group for comparison.

and anti-object groups, the targets rotated with the hand orientation and for the ANOVA we matched up the six directions across orientations so that they had the same end point forces (for a straight line movement). Early and late exposures correspond to the first and last 18 trials in the force field. The final level of adaptation was examined across the final 330 trials (with MPE and force compensation calculated from 270 and 60 trials, respectively). If a significant main effect was found, Tukey's HSD post hoc test was used to examine differences.

The mean MPE and force compensation across subjects during the exposure phase of the experiment were fit with the following exponential functions:

$$
y=\alpha \cdot e^{-\lambda \cdot k}+C
$$

where $C$ is the asymptote of final adaptation, $\lambda$ is the time constant or rate of adaptation, $k$ is the trial number, and $\alpha$ is the magnitude of adaptation. The exponential function was fit using the Matlab function nlinfit, and 95\% confidence intervals (Cls) of the function and the parameters were determined using nlpredci, and nlparci, respectively. To examine the statistical differences between our parameter estimates, we performed block bootstrapping in which we left out each possible set of three participants from each group and fitted the remaining seven. To test whether each parameter varied between the four groups, we generated all possible differences in each parameter from our bootstrap to generate a new bootstrap sample $(120 \times 120$ samples). The six comparisons were then examined at a Bonferroni-adjusted statistical level $(p<$ 0.0083).

\section{Results}

Subjects performed center-out reaching movements to six targets, and were required to match both the position and orientation at the start and end of each reach. For the control group, all movements were made with the same neutral hand orientation (Fig. 1A), whereas for the other three groups, each movement was performed with one of three different hand orientations (neutral position and $\pm 30^{\circ}$; Fig. $1 B$ ). For all groups, movements in the null field were approximately straight (Fig. $3 A-D$, first 240 trials).

For the Cartesian group, the force depended on hand velocity but, critically, did not vary with the hand orientation, and, therefore, the field is unambiguously consistent with forces represented in a Cartesian frame (Fig. 1B). For two of the groups, the entire force depended on the hand orientation, either rotating with the hand (Fig. $1 C$, objectgroup), consistent with the dynamics of a hand-held object, or counter to the hand rotation (Fig. 1D, anti-object).

On the introduction of a force field in the exposure phase, large increases in MPE were observed (Fig. $3 A-D$, gray region) that were not significantly different across the four groups $\left(F_{(3,660)}=0.864 ; p=0.46\right.$; initial exposure). Across trials, the MPE was reduced, and we compared the final levels of adaptation (last 270 trials) using an ANOVA with factors of group, movement direction, and hand orientation. Only the main effect of group was significant $\left(F_{(3,107,400}=620.01 ; p<0.001\right)$, with no significant effect of wrist angle $\left(F_{(2,21,540)}=0.86 ; p=0.42\right)$, movement direction $\left(F_{(6,21,540)}=0.62 ; p=0.69\right)$, or interactions (all $p>0.09$ ). Post hoc tests indicated that the 

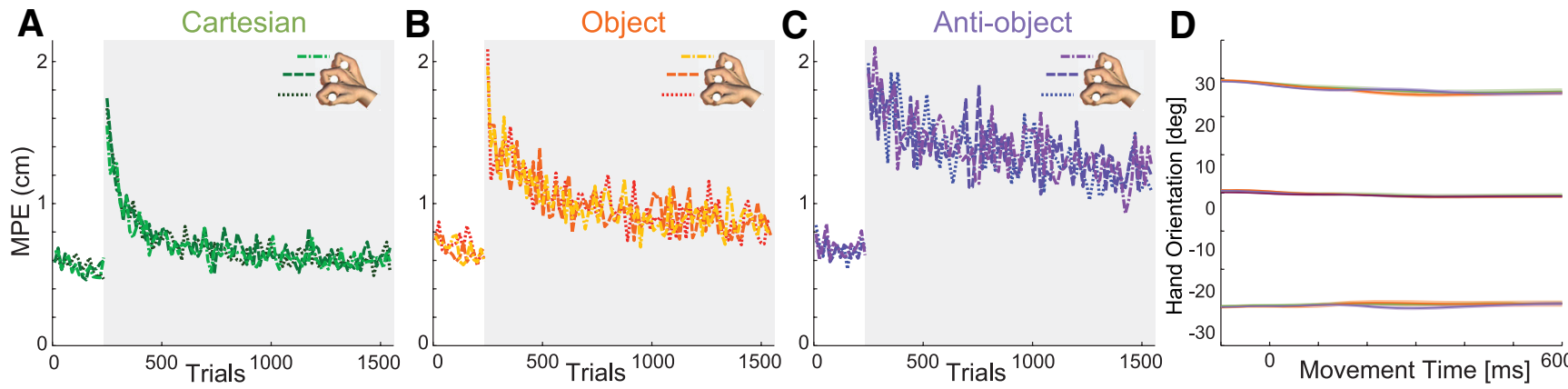

Figure 4. Learning and kinematics as a function of hand orientation. $\boldsymbol{A}-\boldsymbol{C}$, Learning curves separated by hand orientation for three groups. $\boldsymbol{D}$, Hand orientation (mean \pm SE averaged as in Fig. 1) for the four groups as a function of time (colors are as in Fig. 1).

final error for the anti-object group was significantly greater than all others (all $p<0.001)$. The object group had a significantly larger final error than both the Cartesian and control groups $(p<0.001)$. The Cartesian group had a slightly smaller error than the control groups $(p=$ 0.016). Importantly, the learning within each group was similar for each of the hand orientations (Fig. $4 A-C$ ), and subjects were able to control the hand orientation throughout the movements (Fig. 4D).

To examine the feedforward component of learning, independent of any nonspecific changes in arm impedance (Franklin et al., 2003, 2007), we examined the lateral force produced on occasional channel trials (Scheidt et al., 2000; Milner and Franklin, 2005) and calculated the percentage compensation for the force field (Fig. $3 E-H)$. In the pre-exposure phase, the subjects generated minimal force into the channel wall (Fig. $3 E-H$, first 240 trials). At the end of the exposure phase, there was a significant difference in the final force compensation values across the groups $\left(F_{(3,2360)}=72.66 ; p<0.001\right.$, last 60 channel trials, corresponding to the same interval as the 270 trials examined for MPE). Post hoc tests showed that the compensation for the anti-object group was significantly less than all others (all $p<0.001$ ). There were no significant differences between the Cartesian and object groups $(p=$ 0.1 ) or between these two and the control group $(p=0.82$ and $p=0.47$ respectively).

Importantly, our experimental design meant that all groups experienced the same movements and forces for the neutral wrist orientation (Fig. 2). As such, we can directly compare the adaptation that occurred only in this single wrist posture, thereby controlling for both the movement and field experienced (Fig. 5). The results demonstrate that the same findings across all wrist postures (Fig. 3) are also present in this single wrist posture condition.

To compare the rate and final level of learning across all groups, we fit exponential curves to both the kinematic error and force compensation during exposure trials (Fig. 3 , smooth curves show the fits and dashed curve is fits to the control group for comparison). Figure 6 shows the fits for the four groups with $95 \%$ Cls. We used bootstrap analysis (see Materials and Methods) to compare the time constants and asymptotes across the groups. For the kinematic error (Fig. $6 A$ ), the time constants for the control and Cartesian groups were not significantly different from one another $(p=0.38)$ and were significantly shorter than those for either the object or anti-object groups (all $p<$ $0.00001)$. The object group time constant was not different from that for the anti-object group $(p=0.09)$. For the final maximum perpendicular error level (asymptote), the lowest asymptote was seen in the Cartesian and control groups (not significantly different; $p=0.16$ ), while the object group had a significantly higher asymptote (all $p<$ $0.00001)$. Again, the largest asymptote was found for the anti-object group (all $p<0.004$ ).

A similar pattern was found across the parameters determined from fits to the force compensation data (Fig. $6 B)$. For the time constants, there was again no difference between the Cartesian and control groups $(p=0.18)$; however, both groups were significantly faster than the object or anti-object groups (all $p<0.00001$ ). Again, no significant difference was found between the object and anti-object groups after multiple comparison correction $(p$ $=0.03$ ). There were significant differences in the level of final adaptation (asymptote). There was no difference between the control group and the Cartesian group $(p=$ 0.05 ) or the object group ( $p=0.01$ ) after correction; however, there were significant differences between the Cartesian and object groups $(p<0.0001)$. The anti-object group had significantly lower final adaptation than all three groups (all $p<0.00001$ ).

Interestingly, there were strong differences between the results based on the maximum perpendicular error and those based on predictive force compensation. While these two measures of learning normally give similar results, the kinematic error can be reduced independent of predictive force compensation by increasing the stiffness of the limb by cocontraction or increased feedback gains during reaching (Burdet et al., 2001; Franklin et al., 2003, 2012). In our study, while the force compensation results suggested that the object group learned as well as the Cartesian and control groups, the final level of kinematic error measure was still higher. This could indicate that the cocontraction was lower in this object group, but other biomechanical factors related to limb geometry (Burdet et al., 2013) could also have played a role. In either case, the difference in final adaptation for the object group was only on the order of $2 \mathrm{~mm}$. 

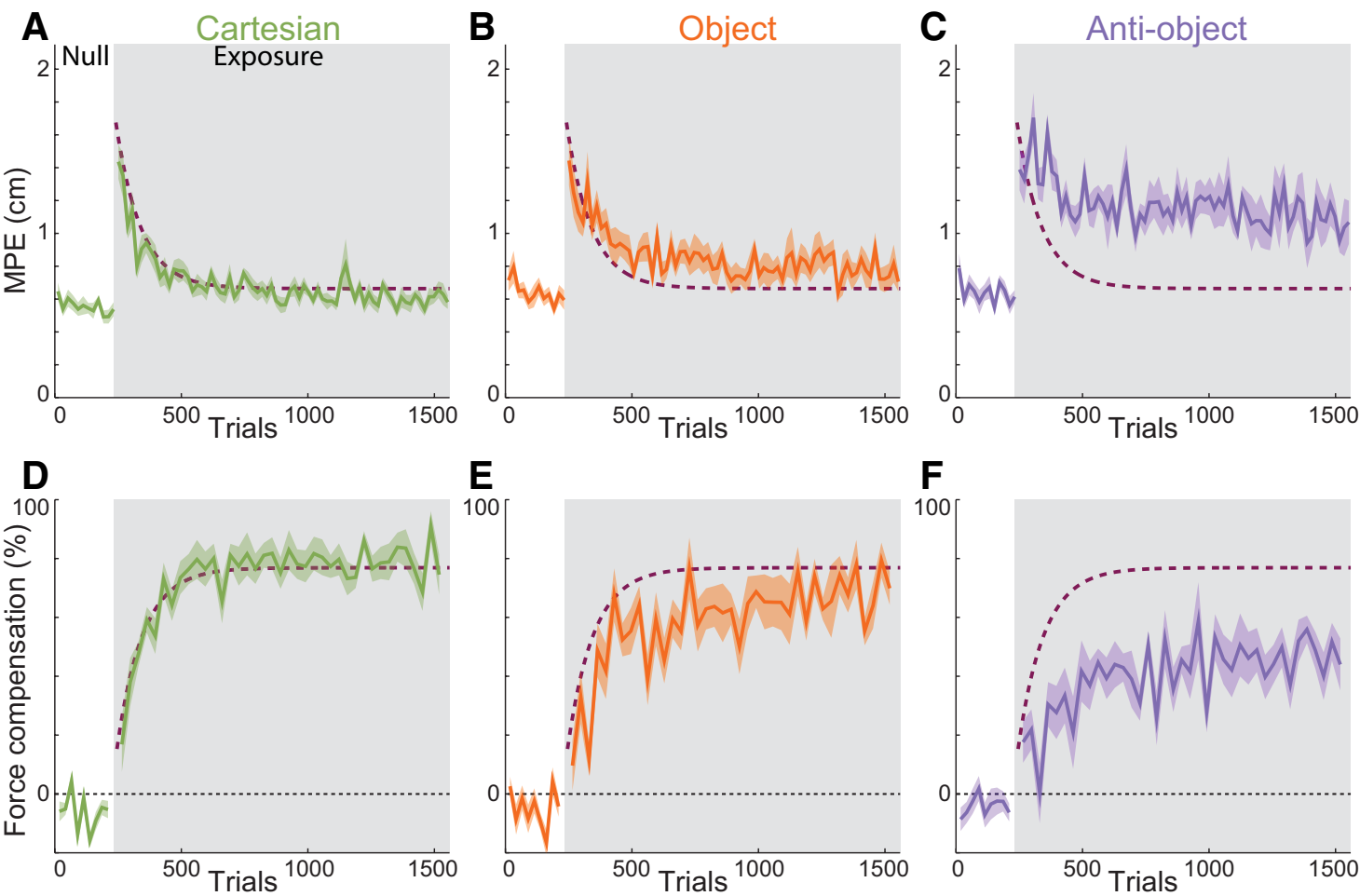

Figure 5. Learning and force compensation for the neutral wrist orientation where the force field is identical across all four groups. $\boldsymbol{A}-\boldsymbol{C}$, The maximum perpendicular error (mean \pm SE across subjects). The dotted line shows the exponential fit for the control group for comparison. $\boldsymbol{D}, \boldsymbol{E}$, Force compensation.

\section{Discussion}

Although early studies have suggested that force field adaptation is learned in intrinsic coordinates (Shadmehr and Mussa-Ivaldi, 1994; Malfait et al., 2002, 2005), our results clearly show that a force field explicitly presented in Cartesian coordinates is learned on a very similar timescale and with levels of learning similar to those of an ambiguous force field (Fig. 3E-H). An ambiguous force field would be learned in the preferred coordinate system (e.g., intrinsic) if such a preferred coordinate system ac- tually exists. In other words, if the dynamic learning is represented in intrinsic coordinates, then we would have expected the Cartesian condition to be more difficult to learn and have a higher final error than the ambiguous field. A similar argument can be made for the field presented in object-based coordinates, where the final force compensation level was not significantly different from that of the Cartesian or control group. Therefore, our results suggest that both the Cartesian group and the object group learned to represent the dynamics in the
A
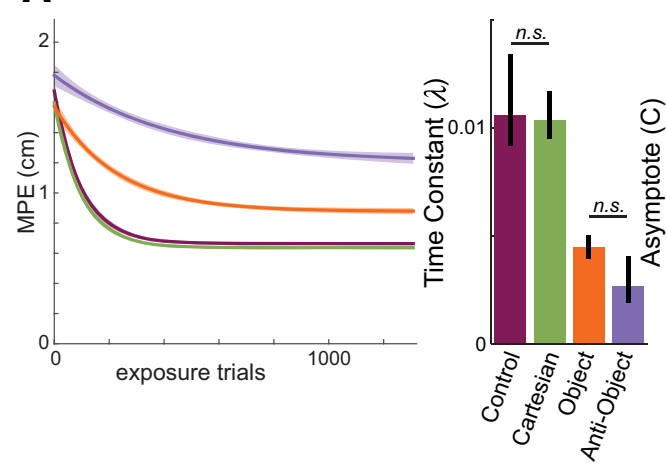

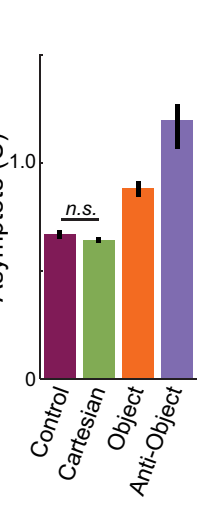

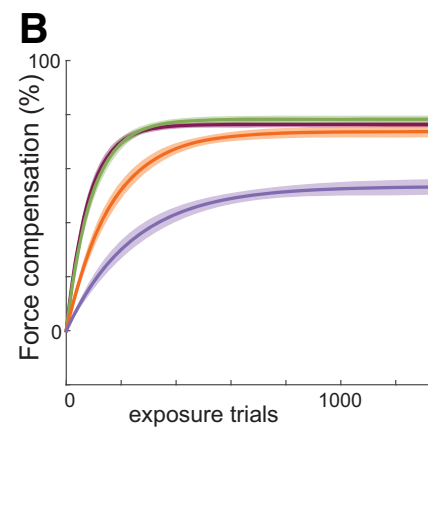

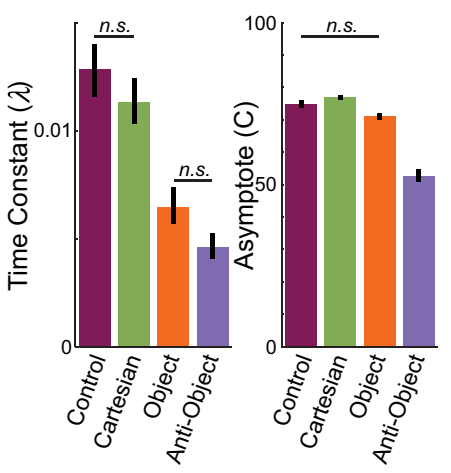

Figure 6. Time constants and asymptotes of learning across conditions. $\boldsymbol{A}$, Left, The mean ( $\pm 95 \% \mathrm{Cl})$ of the exponential fits to the group-averaged MPE for the exposure phase. Right, The asymptote and time constant $( \pm 95 \% \mathrm{Cl})$ of the exponential fits (all comparisons were significant at the $p<0.0083$ level, except for those shown with the flat bar and label $n$.s. indicating that the comparison was not significant after Bonferroni correction). $\boldsymbol{B}$, Fits and parameters for the force compensation data as in $\boldsymbol{A}$. 
coordinate system in which they were presented, demonstrating a flexibility to sculpt the coordinate system when coding dynamics.

We consider the object-based force field as a singular dynamic experienced in three different hand orientations. However, another possibility is that the sensorimotor system represents this as three separate force fields learned independently for each hand orientation. This would place our findings in agreement with those demonstrating that hand orientation (Gandolfo et al., 1996; Yeo et al., 2015) or similar differences in the limb state (Hwang et al., 2003; Howard et al., 2013) can be used to reduce interference and learn opposing force fields, although extending them to three states. However, if this was the case for the object-based force field, then the anti-object-based force field should have been equally easy to learn as the three separate context-dependent fields. Instead, the demonstration that the anti-object force field is not learned equally well shows a limitation in the ability of small changes in hand orientation to allow for independent learning of different force fields over a similar state space. Our results indicate that force field presentations that do not occur naturally are not as quickly or well learned compared with those that can be represented in a more natural coordinate frame.

We propose that ambiguous dynamics are represented in a mixture of coordinate representations (Haswell et al., 2009; Brayanov et al., 2012; Berniker et al., 2014; Parmar et al., 2015), such as Cartesian, intrinsic, and object based, with the weighting of this mixture being driven by the likelihood of each particular representation. Such a mixture would provide a rapid adaptation to the ambiguous force field with the mixing components then appropriately reweighted if the force field became unambiguous (as in the Cartesian and object groups). However, such a mixed representation would struggle to learn representations that could not be achieved through mixing (antiobject).

Our findings shed light onto why so many conflicting results about the nature of coordinate representation were found, such as evidence for both intrinsic (Shadmehr and Mussa-Ivaldi, 1994; Shadmehr and Moussavi, 2000; Malfait et al., 2002, 2005) and extrinsic (Krakauer et al., 2000; Criscimagna-Hemminger et al., 2003; Burgess et al., 2007) generalization. Instead, our work further supports the idea that dynamics can be represented in a mixture of coordinate systems (Haswell et al., 2009; Brayanov et al., 2012; Berniker et al., 2014), of which intrinsic, extrinsic, and object coordinate systems are three of the (possibly many) representations available. Our results jibe with recent neurophysiological studies showing that many neurons show complex mixed selectivity in motor tasks (Churchland et al., 2012) and more cognitive tasks (Mante et al., 2013; Rigotti et al., 2013). Although the results of the anti-object group clearly preclude the possibility that any arbitrary mapping can be learned on a short time scale $(\sim 2 \mathrm{~h})$, a critical question is whether these coordinate representations are fixed a priori or whether they can be learned with sufficient experience.

\section{References}

Aflalo TN, Graziano MSA (2007) Relationship between unconstrained arm movements and single-neuron firing in the macaque motor cortex. J Neurosci 27:2760-2780. CrossRef Medline

Berniker M, Franklin DW, Flanagan JR, Wolpert DM, Kording K (2014) Motor learning of novel dynamics is not represented in a single global coordinate system: evaluation of mixed coordinate representations and local learning. J Neurophysiol 111:1165-1182. CrossRef Medline

Brayanov JB, Press DZ, Smith MA (2012) Motor memory is encoded as a gain-field combination of intrinsic and extrinsic action representations. J Neurosci 32:14951-14965. CrossRef Medline

Burdet E, Osu R, Franklin D, Milner T, Kawato M (2001) The central nervous system stabilizes unstable dynamics by learning optimal impedance. Nature 414:446-449. CrossRef Medline

Burdet E, Franklin DW, Milner TE (2013) Human robotics: neuromechanics and motor control. Cambridge, MA: MIT.

Burgess JK, Bareither R, Patton JL (2007) Single limb performance following contralateral bimanual limb training. IEEE Trans Neural Syst Rehabil Eng 15:347-355. CrossRef Medline

Churchland MM, Cunningham JP, Kaufman MT, Foster JD, Nuyujukian P, Ryu SI, Shenoy KV (2012) Neural population dynamics during reaching. Nature 487:51-56. CrossRef Medline

Criscimagna-Hemminger SE, Donchin O, Gazzaniga MS, Shadmehr R (2003) Learned dynamics of reaching movements generalize from dominant to nondominant arm. J Neurophysiol 89:168-176. CrossRef Medline

Franklin DW, Osu R, Burdet E, Kawato M, Milner TE (2003) Adaptation to stable and unstable dynamics achieved by combined impedance control and inverse dynamics model. J Neurophysiol 90:3270-3282. CrossRef

Franklin DW, Liaw G, Milner TE, Osu R, Burdet E, Kawato M (2007) Endpoint stiffness of the arm is directionally tuned to instability in the environment. J Neurosci 27:7705-7716. CrossRef Medline

Franklin S, Wolpert DM, Franklin DW (2012) Visuomotor feedback gains upregulate during the learning of novel dynamics. J Neurophysiol 108:467-478. CrossRef Medline

Gandolfo F, Mussa-Ivaldi FA, Bizzi E (1996) Motor learning by field approximation. Proc Natl Acad Sci U S A 93:3843-3846. Medline

Graziano MSA (2011) New insights into motor cortex. Neuron 71: 387-388. CrossRef Medline

Haswell CC, Izawa J, Dowell LR, Mostofsky SH, Shadmehr R (2009) Representation of internal models of action in the autistic brain. Nat Neurosci 12:970-972. CrossRef Medline

Hatsopoulos NG (2005) Encoding in the motor cortex: was evarts right after all? Focus on "motor cortex neural correlates of output kinematics and kinetics during isometric-force and arm-reaching tasks." J Neurophysiol 94:2261-2262. CrossRef Medline

Howard IS, Ingram JN, Wolpert DM (2009) A modular planar robotic manipulandum with end-point torque control. J Neurosci Methods 181:199-211. CrossRef Medline

Howard IS, Wolpert DM, Franklin DW (2013) The effect of contextual cues on the encoding of motor memories. J Neurophysiol 109: 2632-2644. CrossRef Medline

Hwang EJ, Donchin O, Smith MA, Shadmehr R (2003) A gain-field encoding of limb position and velocity in the internal model of arm dynamics. PLoS Biol 1:E25. CrossRef Medline

Kakei S, Hoffman DS, Strick PL (1999) Muscle and movement representations in the primary motor cortex. Science 285:2136-2139. Medline

Kalaska JF (2009) From intention to action: motor cortex and the control of reaching movements. Adv Exp Med Biol 629:139-178. CrossRef Medline

Krakauer JW, Pine ZM, Ghilardi MF, Ghez C (2000) Learning of visuomotor transformations for vectorial planning of reaching trajectories. J Neurosci 20:8916-8924. Medline

Malfait N, Shiller DM, Ostry DJ (2002) Transfer of motor learning across arm configurations. J Neurosci 22:9656-9660. Medline

Malfait N, Gribble PL, Ostry DJ (2005) Generalization of motor learning based on multiple field exposures and local adaptation. J Neurophysiol 93:3327-3338. CrossRef Medline 
Mante V, Sussillo D, Shenoy KV, Newsome WT (2013) Contextdependent computation by recurrent dynamics in prefrontal cortex. Nature 503:78-84.

Milner TE, Franklin DW (2005) Impedance control and internal model use during the initial stage of adaptation to novel dynamics in humans. J Physiol (Lond) 567:651-664. CrossRef Medline

Oldfield RC (1971) The assessment and analysis of handedness: the Edinburgh inventory. Neuropsychologia 9:97-113. Medline

Parmar PN, Huang FC, Patton JL (2015) Evidence of multiple coordinate representations during generalization of motor learning. Exp Brain Res 233:1-13. CrossRef Medline

Rigotti M, Barak O, Warden MR, Wang X-J, Daw ND, Miller EK, Fusi $S$ (2013) The importance of mixed selectivity in complex cognitive tasks. Nature 497:585-590. CrossRef Medline

Scheidt RA, Reinkensmeyer DJ, Conditt MA, Rymer WZ, MussaIvaldi FA (2000) Persistence of motor adaptation during con- strained, multi-joint, arm movements. J Neurophysiol 84:853-862. Medline

Scott SH (2008) Inconvenient truths about neural processing in primary motor cortex. J Physiol (Lond) 586:1217-1224. CrossRef Medline

Shadmehr R, Mussa-Ivaldi FA (1994) Adaptive representation of dynamics during learning of a motor task. J Neurosci 14:32083224. Medline

Shadmehr R, Moussavi ZM (2000) Spatial generalization from learning dynamics of reaching movements. J Neurosci 20:7807-7815. Medline

Smith MA, Ghazizadeh A, Shadmehr R (2006) Interacting adaptive processes with different timescales underlie short-term motor learning. Plos Biol 4:e179. CrossRef Medline

Yeo SH, Wolpert DM, Franklin DW (2015) Coordinate representations for interference reduction in motor learning. PLoS One 10: e0129388. CrossRef Medline 